VoL. $76(2007)$ [111-132]

\title{
FINITE MATRIX MODEL OF QUANTUM HALL FLUIDS ON $S^{2}$
}

\author{
Yi-Xin Chen, Mark D. Gould and Yao-Zhong Zhang
}

\begin{abstract}
Based on Haldane's spherical geometrical formalism of the two-dimensional quantum Hall fluids, the relation between the noncommutative geometry of $S^{2}$ and the two-dimensional quantum Hall fluids is exhibited. A finite matrix model on the twosphere is explicitly constucted as an effective description of the fractional quantum Hall fluids of finite extent, and the complete sets of physical quantum states of this matrix model are determined. We also describe how the low-lying excitations in the model are constructed in terms of the quasi-particle and quasi-hole excitations. It is shown that there exists a Haldane hierarchical structure in the two-dimensional quantum Hall fluid states of the matrix model. These hierarchical fluid states are generated by the parent fluid state by condensing the quasi-particle and quasi-hole excitations level by level.
\end{abstract}

\section{INTRODUCTION}

The planar coordinates of quantum particles in a constant magnetic field provide a natural realisation of noncommutative space [7]. The physics of electrons in the lowest Landau level exhibits many fascinating properties. In particular, when the electron density equals to some rational fraction of the density corresponding to a fully filled lowest Landau level, the electrons are condensed into special incompressible fluid states whose excitations exhibit unusual phenomena such as fractional charges and fractional statistics. For the filling fractions $\nu=1 / m$, the physics of these states is accurately described by the wave functions proposed by Laughlin [13].

Recently, an interesting connection between the quantum Hall effect and noncommutative field theory has been revealed in the literature. In particular, Susskind [19] proposed that the noncommutative Chern-Simons theory on a plane may provide a description of the (fractional) quantum Hall fluid and specifically of the Laughlin states. This noncommutative Chern-Simons theory describes a spatially infinite quantum Hall

Received 5th December, 2006

This work was partly supported by Austrialian Research Council. The work of Chen was also partly supported by the NNSF of China (Grant No.90503009), and the Tang Yong-Qian Foundation of Zhejiang University.

Copyright Clearance Centre, Inc. Serial-fee code: 0004-9727/07 \$A2.00+0.00. 
system. It gives the Laughlin states at filling fractions $\nu$ when the number of electrons confined in the lowest Landau level is infinitely large. The fields of this theory are infinite matrices which act on an infinite Hilbert space, to account for an infinite number of electrons. Subsequently, Polychronakos [17] proposed a regularised matrix version of the noncommutative Chern-Simons theory in an effort to describe a finite system with a finite number of electrons in limited spatial extent. This matrix model was shown to reproduce the basic properties of the quantum Hall droplets. Furthermore, it was shown that there exists a complete minimal basis for the exact wave functions of the regularised matrix theory at arbitrary level $\nu^{-1}$ and rank $N$, and these wave functions are in one to one correspondence with the Laughlin wave functions describing the excitations of the quantum Hall droplet composed of $N$ electrons at filling fraction $\nu[10]$. It is believed that the matrix model is equivalent to the theory of composite fermions in the lowest Landau level, and should provide an accurate description of the fractional quantum Hall states. However, although the long distance behaviours of the two theories mentioned above agree, their short distance behaviours are different [12]. It should also be pointed out that the finite matrix model proposed by Polychronakos is still defined on a two-dimensional plane.

It is well known that it is more convenient to formulate the quantum Hall system on a two-dimensional sphere. Such a formulation appeared in the work of Haldane on the fractional quantum Hall effect [9]. The Haldane model deals with a two-dimensional gas of $N$ electrons on a spherical surface in a radial monopole magnetic field. The Dirac monopole lies at the centre of the two-dimensional sphere. This model describes not only a variant of the Laughlin model with translationally invariant wave functions, but also a hierarchy of the quantum Hall fluid states. In certain limits, the Haldane model exhibits a clear connection with the noncommutative Chern-Simons theory. Precisely, the noncommutative property of the particle coordinates in the Haldane model is described by a fuzzy two-sphere [15] ( see below for details ). It is believed that different noncommutative manifolds correspond to finite matrix models with different geometrical properties. Recently, there has been much interest in formulating Chern-Simons theories on various noncommutative manifolds $[16,14,2]$.

Based on the work of Hellerman and Raamsdonk [10] on the equivalence of twodimensional quantum Hall physics and noncommutative field theory, one knows that a secondly-quantised field theoretical description of the quantum Hall fluids for various filling fractions should be provided by some noncommutative field theory. On a 2-dimensional plane, such a noncommutative field theory is the regularised matrix $U(1)$ noncommutative Chern-Simons theory. The aim of this paper is to construct a finite matrix model which provides the description of the quantum Hall system on $S^{2}$. Moreover, we shall explore the possible hierarchical structure of the quantum Hall fluids in the finite matrix model. 
This paper is organised as follows. Section two introduces the two-dimensional quantum Hall model on $S^{2}$ proposed by Haldane [9] and analyses the non-commutativity of the $S^{2}$ coordinates by focusing on the lowest Landau level state of the system. It will be shown that the noncommutative space has the geometry of the fuzzy $S^{2}$. We introduce the Hopf mapping for the fuzzy $S^{2}$, and construct a regularised effective matrix theory on it. This matrix theory is singularity-free in the field configurations obtained by the Hopf fibration with the fuzzy $S^{2}$ as the base. These configurations are described by spinors with two complex components. Taking the number of particles infinitely large, we show that the effective matrix theory is equivalent to the $U(1)$ noncommutative ChernSimons theory proposed by Susskind. However, our matrix model is different from the one proposed by Polychronakos: unlike the Polychronakos model, matrix fields in our effective theory are complex two-component spinors since the theory is invariant under the $U(1)$ gauge transformation of such spinors. In section three, we analyse the Fock space structure of our matrix model and determine its complete sets of physical quantum states. Section four investigates a condensation mechanism for the low-lying excitations in our finite matrix model of the quantum Hall fluids on $S^{2}$. It is shown that there exists a Haldane hierarchy in the matrix model and such hierarchy is dynamically generated by condensing the excitations of the quantum Hall fluids level by level. Section five inciudes a summary on our main results and some remarks on further research in the direction.

\section{HaLdane's QUANTUM Hall SYSTEM AND FUZzy $S^{2}$ STRUCTURE}

In the quantum Hall effect problem, it is advantageous to consider a compact spherical space which can be mapped to the flat Euclidean space by the standard stereographic mapping [9]. Haldane considered a system, in which a two-dimensional electron gas of $N$ particles is placed on a two-sphere $S^{2}$ in a radial Dirac monopole magnetic field $B$. A point $x^{a}$ on $S^{2}$ with radius $R$ can be described by the dimensionless vector coordinates $n^{a}=x^{a} / R, a=1,2,3$ which satisfy $n^{a} n^{a}=1$. Here and throughout, we shall often use the usual summation convention, that is, repeated indices are understood to be summed. The single particle Hamiltonian in this system reads

$$
\mathcal{H}=\frac{1}{2 M R^{2}} \sum_{a} \Lambda^{a} \Lambda^{a}
$$

where $M$ is the effective mass, and $\vec{\Lambda}=\vec{r} \times[-i \hbar \vec{\nabla}-e \vec{A}]=\vec{r} \times[\vec{p}-e \vec{A}]$ is the dynamical angular momentum of the particle. The relation between the vector potential $\vec{A}$ and the magnetic field is given by $\vec{\nabla} \times \vec{A}=B \vec{n}$. Due to the presence of the Dirac monopole field, the dynamical angular momentum $\Lambda^{a}$ does not obey the algebraic relation of the usual angular momentum. One can easily check that they satisfy the commutation relations

$$
\left[\Lambda^{a}, \Lambda^{b}\right]=i \hbar \varepsilon^{a b c}\left(\Lambda^{c}+e B R^{2} n^{c}\right)
$$


However, $L^{a} \equiv \Lambda^{a}-e B R^{2} n^{a}$ provide the generators of the rotations in the presence of the Dirac monopole field. Indeed, by direct calculation, one can show that

$$
\left[L^{a}, L^{b}\right]=i \hbar \varepsilon^{a b c} L^{c}, \quad\left[L^{a}, \Lambda^{b}\right]=i \hbar \varepsilon^{a b c} \Lambda^{c}, \quad\left[L^{a}, n^{b}\right]=i \hbar \varepsilon^{a b c} n^{c} .
$$

The vector $\vec{\Lambda}$ has no component normal to the surface and thus we have $L^{a} n^{a}=-e B R^{2}$ $=n^{a} L^{a}$. As pointed out by Haldane [9], the spectrum of $\Lambda^{a} \Lambda^{a}$ determined by the angular momentum operators $L^{a}$ is $\Lambda^{a} \Lambda^{a}=\left(L+e B R^{2}\right)^{a}\left(L+e B R^{2}\right)^{a}=\hbar^{2}\left[l(l+1)-S^{2}\right]$. Because $\vec{\Lambda}$ is a hermitian operator and the Hamiltonian $H \sim \Lambda^{a} \Lambda^{a}$ must be larger than or equal to zero, one determines that $l=S+n, n=0,1,2, \ldots$. Hence, for a given $S$, the energy eigenvalues of the Hamiltonian (1) are

$$
E_{n}=\frac{\hbar^{2}}{2 M R^{2}}[n(n+1)+(2 n+1) S]
$$

The above energy spectrum when $n=0$ corresponds to the lowest Landau level. Since $S$ is the spin of the particle, the degeneracy of the lowest Landau level is $2 S+1$.

On the other hand, we can discuss the classical canonical dynamics of the Hamiltonian $H$ and $H+V\left(x^{a}\right)$, where $V\left(x^{a}\right)$ is the potential energy with rotational symmetry. By means of the correspondence between classical and quantum physics, one can straightforwardly read off the fundamental Poisson brackets of the classical degrees of freedom from their corresponding commutation relations. In the canonical Hamiltonian formulation, the evolution of the dynamical variables with time is described by the canonical Hamilton equation, that is, $\dot{\Lambda}^{a}=\left\{\Lambda^{a}, H\right\}=(e B) / M \varepsilon^{a b c} \Lambda^{b} n^{c} \neq 0$. This implies that the dynamical angular momentum is not a conservative quantity of the system. In fact, in the presence of the Dirac monopole, the generator of the rotations is modified to $L^{a}$, which is a conservative quantity since $\dot{L}^{a}=\left\{L^{a}, H\right\}=(-1) /\left(M R^{2}\right) \varepsilon^{a b c} \Lambda^{b} \Lambda^{c}=0$. If we consider the system with the rotationally symmetric potential $V\left(x^{a}\right), L^{a}$ is still conservative. That is

$$
\dot{L}^{a}=\dot{\Lambda}^{a}-e B R^{2} \dot{n}^{a}=0 .
$$

The variation of $n^{a}$ with time is given by the canonical Hamiltonian equation of $\Lambda^{a}$

$$
\dot{n}^{a}=\frac{1}{e B R^{2}}\left[\frac{e B}{M} \varepsilon^{a b c} n^{b} \Lambda^{c}+\frac{\partial V}{\partial n^{b}} \varepsilon^{a b c} n^{c}\right] .
$$

Since we are interested in the equation of motion in the lowest Landau level, we take the infinite limit of mass $M \rightarrow \infty$. In this limit, we obtain the following equation of motion

$$
\dot{n}^{a}=\frac{1}{e B R^{2}} \frac{\partial V}{\partial n^{b}} \varepsilon^{a b c} n^{c} \text {. }
$$

This implies that the momentum variables can be fully eliminated in the lowest Landau level. The elimination of momentum variables leads to the coordinates on the two-sphere 
which are noncommutative. Restricted to the lowest Landau level state, the equation of motion may be equivalently derived from the fundamental Poisson bracket

$$
\left\{n^{a}, n^{b}\right\}=\frac{1}{e B R^{2}} \varepsilon^{a b c} n^{c}, \quad n^{a} n^{a}=1 .
$$

This Poisson algebra can be realised by the matrix commutator

$$
\left[n^{a}, n^{b}\right]=\frac{1}{e B R^{2}} i \varepsilon^{a b c} n^{c}, \quad n^{a} n^{a}=1 .
$$

In conclusion, if we focus on the lowest Landau level of the system, the two-dimensional spherical geometry becomes the geometry of the fuzzy $S^{2}$ which is noncommutative [15].

In order to exhibit the fuzzy property of algebra (9), we take an isomorphic mapping from (9) to algebra $S U(2)$. Set $n^{a} \mapsto\left(X^{a}\right) /\left(e B R^{2}\right)$. Then equation (9) becomes

$$
\left[X^{a}, X^{b}\right]=i \varepsilon^{a b c} X^{c}
$$

which is nothing but the standard $S U(2)$ algebra. The quadratic Casimir of $S U(2)$ in the $N$-dimensional irreducible representation is given by

$$
X^{a} X^{a}=\frac{1}{4}\left(N^{2}-1\right)
$$

The constraint $n^{a} n^{a}=1$ leads to

$$
e B R^{2}=\sqrt{\frac{1}{4}\left(N^{2}-1\right)} .
$$

This relation implies that the two parameters $B$ and $R$ are quantised, exhibiting the property of the fuzzy two-sphere. In order to compare with the usual expression, we rewrite $e B R^{2}$ as $e B R \cdot R \equiv R /\left(\theta^{\prime}\right)=\sqrt{\left(N^{2}-1\right) / 4}$, where $\theta^{\prime}=e B R$. Then, we have

$$
\left[n^{a}, n^{b}\right]=i \frac{\theta^{\prime}}{R} \varepsilon^{a b c} n^{c} \equiv i 2 \theta \varepsilon^{a b c} n^{c}, \quad n^{a} n^{a}=1 .
$$

This algebraic relation is the starting point of the following discussions about the Hopf mapping of the fuzzy $S^{2}$.

It is well known that in the appearance of a monopole field one can not define a vector potential which is singularity-free on the entire manifold $S^{2}$. The use of two vector potentials living respectively on the north and the south semi-spheres, which was advocated by $\mathrm{Wu}$ and Yang [20], provides a way of solving the singularity problem. However, the Wu-Yang procedure is not well adapted for our later purpose. Thus in this paper we shall use the formalism proposed by Balachandran in [3]. This formalism allows one to obtain the singularity-free effective Lagrangian. The key step is to carry out the first Hopf fibration of $S^{2}$ to get $S^{3}$. The first Hopf map is a mapping from $S^{3}$ to $S^{2}$. In the presence of a Dirac monopole, the $U(1)$ bundle over $S^{2}$ is topologically 
non-trivial. However, one can use the first Hopf map to define a non-singular vector potential everywhere on $S^{3}$. This procedure is called the first Hopf fibration.

Let us use the notation $z=\left(\begin{array}{l}z_{1} \\ z_{2}\end{array}\right)$ for the two-component complex spinor $z$. This complex spinor $z$ has three degrees of freedom since the normalisation condition $z^{\dagger} z=$ $1=\left|z_{1}\right|^{2}+\left|z_{2}\right|^{2}$ is the only constraint on the two complex numbers $z_{1}, z_{2}$. So they are actually defined on a $S^{3}$ surface. The Hopf projection map which takes us from $S^{3}$ to $S^{2}$ is given by

$$
\vec{n}=z^{\dagger} \vec{\sigma} z
$$

where $\sigma^{a}$ are the three Pauli matrices. It should be noticed that the $U(1)$ transformation $z \rightarrow e^{i \alpha} z$ leaves $n^{a}$ invariant and so the inverse image of any point on $S^{2}$ is a circle on $S^{3}$. Now we ask what Poisson relation for $z$ 's can be used to produce the Poisson algebra (8) of the fuzzy $S^{2}$. It can be easily checked that the answer to this question is

$$
\left\{z, z^{\dagger}\right\}=\theta,
$$

that is,

$$
\left\{z_{1}, \bar{z}_{1}\right\}=\theta=\left\{z_{2}, \bar{z}_{2}\right\},\left\{z_{1}, \bar{z}_{2}\right\}=0=\left\{z_{2}, \bar{z}_{1}\right\}
$$

Subsequently, we focus on the description of the effective action of particles in the presence of a Dirac monopole field. Due to the existence of the monopole, the $U(1)$ bundle over $S^{2}$ is topologically non-trivial. This leads to the appearance of an additional term, called the Wess-Zimino term, in the effective action of the system. Such an effective action had been obtained by Stone in [18], which has the form

$$
I=\frac{1}{2 f} \int d t n^{a} n^{a}+\int \mathcal{A}^{a} d n^{a},
$$

where $f$ is dependent on the parameters of the system, and $\mathcal{A}$ is the potential of the Dirac monopole which cannot be globally expressed on $S^{2}$ due to the singularity of the Dirac string. However, by means of the Hopf fibration of $S^{2}$ and its $U(1)$ gauge symmetry, the monopole potential can be globally defined on $S^{3}$ as

$$
\mathcal{A}=i \frac{\tilde{\lambda}}{2}\left[z^{\dagger} d z-d z^{\dagger} z\right]
$$

where $\tilde{\lambda}$ is related to the magnetic charge of the Dirac monopole. It should be pointed out that the potential $\mathcal{A}$ is equivalent to $\mathcal{A}^{a} d n^{a}$ up to a $U(1)$ gauge transformation, and is non-singular everywhere on $S^{3}$. Furthermore, the first term in the effective action (17) can also be described by the spinors defined on $S^{3}[3,1]$. In fact, after quantising the effective action in which the Hopf fibration has been carried out, one gets the energy 
spectrum of the Hamiltonian (1) [1]. Hence, the quantum Hall system on the spherical geometry as discussed by Haldane can be equivalently described by the effective action (17) with the Hopf fibration potential $\mathcal{A}$ (18). Restricted to the lowest Landau level state, the contribution from the kinetic energy in the effective action can be ignored, which is equivalent to taking an infinite limit of $f$. So the physics in the lowest Landau level is described by the action

$$
I_{e}=\int \mathcal{A}
$$

As mentioned above, the $U(1)$ gauge transformation $z \rightarrow e^{i \alpha} z$ leaves $n^{a}$ invariant, so the effective action after the Hopf fibration is also invariant under such a $U(1)$ gauge transformation. Thus, projected to the lowest Landau level state, the effective action becomes $I_{e}=i \widetilde{\lambda} / 2 \int d t\left[z^{\dagger} \partial_{t} z-\partial_{t} z^{\dagger} z\right]$ which should possess the $U(1)$ gauge symmetry. By the standard way of introducing the coupling of a gauge field, we can write the effective action in the explicitly gauge invariant form $I_{e}=i(\widetilde{\lambda}) / 2 \int d t\left[z^{\dagger}\left(\partial_{t}+i A_{0}\right) z-\left(\partial_{t}-i A_{0}\right) z^{\dagger} z\right]$, where $A_{0}$ is a $U(1)$ gauge field. Indeed, this action is invariant under the $U(1)$ gauge transformations $z \rightarrow e^{i \alpha} z$ and $A_{0} \rightarrow A_{0}-\partial_{t} \alpha$. However, now the spinor $z$ becomes noncommutative since it arises from the Hopf mapping of the fuzzy $S^{2}$. The matrix realisation of $z$ is required due to the non-trivial algebraic relations (15) [that is (16)]. The gauge field $A_{0}$ should act on the matrix $z$ by adjoint operation in order for the covariant derivative $\partial_{t}+i A_{0}$ to have the derivative property. Finally, the effective action projected to the lowest Landau level state is given by

$$
I_{e}=i \frac{\tilde{\lambda}}{2} \int d t \operatorname{Tr}\left\{z^{\dagger}\left(\partial_{t} z+\left[A_{0}, z\right]\right)-\left(\partial_{t} z^{\dagger}-\left[A_{0}, z^{\dagger}\right] z\right\}\right.
$$

This matrix theory is similar to that describing D0-branes in the string theory $[4,5]$. We can use this theory to investigate the fluctuations of the spherical brane, which describes the excitations of the Hall fluids on $S^{2}$, by expanding the matrix field in terms of the fluctuations around the classical configurations [5].

First of all, let us introduce $\xi^{r}, r=1,2$, as the parameterising coordinates of the $S^{2}$. The transformations of area preserving diffeomorphisms on this two-dimensional space are given by

$$
\xi^{r} \rightarrow \xi^{r}+\beta^{r}(\xi), \quad \partial_{r}\left(w(\xi) \beta^{r}(\xi)\right)=0,
$$

where $\beta^{r}$ can be locally written as

$$
\beta^{r}(\xi)=\frac{\varepsilon^{r s}}{w(\xi)} \partial_{s} \beta(\xi)
$$

and $w(\xi)$ is a 2-dimensional measure for the normalisation. The transformation rules of the fields are determined by introducing the following Poisson brackets

$$
\{A, B\}=\frac{\varepsilon^{r s}}{w(\xi)} \partial_{r} A \partial_{s} B
$$


Then the transformations of the fields are $\delta X^{a}=\left\{\beta, X^{a}\right\}$ and $\delta A=\partial_{t} \beta+\{\beta, A\}$. The coordinates $\xi^{r}, r=1,2$, parameterise not only the fields $n^{a}$ on $S^{2}$ but also the spinor field $z$ through the Hopf mapping $\vec{n}=z^{\dagger} \vec{\sigma} z$. However, in order to describe the dynamics of the system consistently, the definition of the Poisson bracket (23) should coincide with that of the fundamental Poisson bracket (15). Comparing (23) with (15), we obtain

$$
W(\xi)=\frac{2}{\theta} \operatorname{det}\left|\frac{\partial\left(x^{1}, x^{2}\right)}{\partial\left(\xi^{1}, \xi^{2}\right)}\right|=\frac{2}{\theta} \operatorname{det}\left|\frac{\partial\left(x^{3}, x^{4}\right)}{\partial\left(\xi^{1}, \xi^{2}\right)}\right| \equiv \theta^{-1} W,
$$

where $z_{1}=x^{1}+i x^{2}$ and $z_{2}=x^{3}+i x^{4}$. According to the transformation rule of the fields, we have

$$
\delta x^{i}=\frac{\theta}{W} \varepsilon^{r s} \partial_{r} \beta \partial_{s} x^{i}=\frac{\theta}{W} \varepsilon^{r s} \partial_{j} \beta \partial_{r} x^{j} \partial_{s} x^{i}=\theta \varepsilon^{i j} A_{j},
$$

and

$$
\delta x^{\tilde{i}}=\frac{\theta}{W} \varepsilon^{r s} \partial_{r} \beta \partial_{s} x^{\tilde{i}}=\frac{\theta}{W} \varepsilon^{r s} \partial_{\tilde{j}} \beta \partial_{r} x^{\tilde{j}} \partial_{s} x^{\tilde{i}}=\theta \varepsilon^{\widetilde{i j}} A_{\tilde{j}},
$$

where $i, j=1,2$ and $\tilde{i}, \tilde{j}=3,4$. It should be understood that in the above transformations the matrix variables are expanded in terms of the fluctuations $A$ around the classical solutions $x^{i(0)}$ and $x^{\tilde{i}(0)}$, which determine the classical spinor solution $z^{(0)}$. These classical solutions $z^{(0)}$ and $z^{\dagger(0)}$ obey the fundamental Poisson relation (15). Substituting the matrix variable expansions with fluctuations into the effective action $I_{e}$, we get

$$
\begin{aligned}
I_{e}=\tilde{\lambda} \int d t \operatorname{Tr}\left\{2 \theta A_{0}\right. & +\theta^{2} \varepsilon^{\mu \nu \lambda}\left(A_{\mu} \partial_{\nu} A_{\lambda}+\frac{2}{3} A_{\mu} A_{\nu} A_{\lambda}\right) \\
& \left.+\theta^{2} \varepsilon^{\overline{\mu \nu} \tilde{\lambda}}\left(A_{\tilde{\mu}} \partial_{\tilde{\nu}} A_{\tilde{\lambda}}+\frac{2}{3} A_{\tilde{\mu}} A_{\tilde{\nu}} A_{\tilde{\lambda}}\right)\right\} .
\end{aligned}
$$

The quantities $x^{i(0)}$ and $x^{i(0)}$ are matrices of the classical solution to be related with the noncommutative coordinates of the fuzzy $S^{2}$ by means of the Hopf mapping. Since any matrix can be expressed in terms of a finite sum of the products $\prod_{i \tilde{i}} \exp \left\{i p_{i} x^{i(0)}\right\} \exp \left\{i p_{i} x^{b(0)}\right\}$, the $N \times N$ matrices $A_{\mu}$ and $A_{\tilde{\mu}}$ can be thought of as functions of $x^{i(0)}$ and $x^{\tilde{i}(0)}$. Based on this fact, we can derive the effective Lagrangian in the continuum limit by taking $N$ large. The changes of the coordinates $\xi^{r}, r=1,2$ parameterising the spherical geometry induce the variations of the matrix fields $x^{i(0)}$ and $x^{\tilde{i}(0)}$. In the continuum limit, the $N \times N$ matrices $A_{\mu}^{a b}$ are mapped to smooth functions of the noncommutative coordinates $x^{i(0)}$ and $x^{\tilde{i}(0)}$. For such fields, we can introduce the Weyl ordering to define a suitable ordering for their products in the effective Lagrangian. This implies that the ordinary product is replaced by the noncommutative $\star$-product. Here, the transition rules from fields on the noncommutative space to ordinary functions with the star-product are

$$
[f, g] \rightarrow i \frac{\theta}{W} \varepsilon^{r s} \partial_{r} f \partial_{s} g, \quad \operatorname{Tr}\left(f_{1} \cdots f_{n}\right) \rightarrow \frac{W}{2 \pi \theta} \int\left(f_{1} \star \cdots \star f_{n}\right) .
$$


Thus the effective action describing the fluctuations is

$$
I_{e}=\int d^{3} \xi A_{0} J^{0}+\frac{\lambda \theta}{4 \pi} \int d^{3} \xi \varepsilon^{r s t}\left(A_{r} \star \partial_{s} A_{t}+\frac{2}{3} A_{r} \star A_{s} \star A_{t}\right),
$$

where $\lambda=4 \widetilde{\lambda} W$ and $J^{0}=\lambda / 2$.

The first term in the above equation is the chemical potential and the second term is the standard action for the $U(1)$ noncommutative Chern-Simons theory. Susskind [19] proposed this theory as the description of the quantum Hall fluids on the plane. This Chern-Simons theory necessarily describes an infinite quantum Hall system since the non-commutativity condition requires an infinite dimensional Hilbert space. In other words, the fields in this theory are infinite matrices. However, it is well known that Haldane's description of the quantum Hall effect on a sphere is equivalent to that of Laughlin's on a plane in the thermodynamic limit, taking the number of electrons $N$ large. Our conclusion is that in large $N$, the quantum Hall system on the two-sphere is also described by the $U(1)$ noncommutative Chern-Simons theory. Physically, such a conclusion is reasonable.

However, if we want to describe the quantum Hall fluids of a finite number of electrons on the sphere, we must regularise the noncommutative Chern-Simons theory describing an infinite number of electrons. By means of the Hopf fibration, the spinor $z=\left(\begin{array}{l}z_{1} \\ z_{2}\end{array}\right)$ can be used to describe the dynamics of the electrons on $S^{2}$. So, unlike the finite matrix model proposed by Polychronakos in [17], the regularised matrix model for particles on $S^{2}$ should correspond to the $U(1)$ noncommutative Chern-Simons theory with spinor matrix fields. It should be pointed out that in such a model, the spinor $z$ must be regarded as a field with a single particle rather than that with two particles $z_{1}$ and $z_{2}$.

\section{Regularised $U(1)$ Noncommutative CheRn-Simons theory on $S^{2}$}

In this section we analyse the regularised version of the $U(1)$ noncommutative ChernSimons theory on $S^{2}$. This regularised matrix model should recover the $U(1)$ noncommutative Chern-Simons model on the plane in the large $N$ limit. Explicitly, in the large $N$ limit, the equation of motion for $A_{0}$ provides the non-commutativity of the coordinates, which leads to the classical matrix commutator (9). Such a regularised matrix model associated with the spinor matrix field $z$ can be obtained by following the construction of Polychronakos for the finite matrix model on the plane [17]. Notice that the Hopf mapping makes the normal vector on $S^{2}$ be related to the coordinates of $S^{3}$ described by the two-component spinor $z=\left(\begin{array}{l}z_{1} \\ z_{2}\end{array}\right)$, that is, $n^{a}=z^{\dagger} \sigma^{a} z$. This mapping relation is invariant under the $U(1)$ gauge transformation $z \rightarrow z^{\prime}=e^{i a}\left(\begin{array}{l}z_{1} \\ z_{2}\end{array}\right)$. So it is natural for us 
to propose the following action

$$
S_{p}=\frac{\lambda}{4} \int d t \operatorname{Tr}\left\{i Z^{\dagger} D_{t} Z+2 \theta A_{0}-\omega Z^{\dagger} Z\right\}+\frac{1}{2} \Psi^{\dagger}\left(i \dot{\Psi}-A_{0} \Psi\right)+\text { h.c. }
$$

to describe the system with finite number of electrons living on the two-dimensional sphere, where the covariant derivative is defined as $D_{t}=\partial_{t}+i\left[A_{0}, \quad\right]$. In the above equation, $\Psi$ and $Z$ are spinors with two components, defined by $\Psi=\left(\begin{array}{l}\Psi_{1} \\ \Psi_{2}\end{array}\right)$ and $Z=\left(\begin{array}{l}Z_{1} \\ Z_{2}\end{array}\right)$, respectively. $Z_{\alpha}, \alpha=1,2$ are $N \times N$ complex matrices, $A_{0}$ is a $N \times N$ hermitian matrix, and $\Psi_{\alpha}, \alpha=1,2$ are complex $N$-vectors. They, in the fundamental representation of the gauge group $U(N)$, are transformed as

$$
Z_{\alpha} \rightarrow U Z_{\alpha} U^{-1}, \quad \Psi_{\alpha} \rightarrow U \Psi_{\alpha}, \quad A_{0} \rightarrow U A_{0} U^{-1}+i \dot{U} U^{-1} .
$$

It is obvious that the action $S_{p}$ is invariant under the $U(N)$ gauge transformation. Thus we can choose a gauge for $A_{0}$ and impose the equation of motion of $A_{0}$ as the constraint

$$
\frac{\lambda}{2}\left[Z, Z^{\dagger}\right]+\Psi \Psi^{\dagger}=\lambda \theta \text {. }
$$

If we re-scale $Z$ to $\sqrt{2 / \lambda} Z^{\prime}$ and denote $Z^{\prime}$ as $Z$, the above equation can be rewritten as

$$
\left[Z, Z^{\dagger}\right]+\Psi \Psi^{\dagger}=\lambda \theta
$$

One sees from the action (28) that the conjugate momenta of $Z$ and $\Psi$ are $Z^{\dagger}$ and $\Psi^{\dagger}$, respectively. So they obey the classical matrix commutators

$$
\left[\left(Z_{\alpha}\right)_{m n},\left(Z_{\beta}^{\dagger}\right)_{k l}\right]=-i \delta_{m k} \delta_{n l} \delta_{\alpha \beta} \text { and }\left[\left(\Psi_{\alpha}\right)_{m},\left(\Psi_{\beta}^{\dagger}\right)_{n}\right]=-i \delta_{m n} \delta_{\alpha \beta}
$$

Since the spinor describes a particle moving on the two-sphere, we should regard such spinor as a single oscillator. So there are $N^{2}+N$ uncoupled oscillators in the present system. Their Hamiltonian is

$$
H=\omega \operatorname{Tr} Z^{\dagger} Z=\omega \sum_{m, n, \alpha}\left(Z_{\alpha}^{\dagger}\right)_{m n}\left(Z_{\alpha}\right)_{n m}
$$

The constraint equation can be used to reduce the space of the quantum physical states. Since the constrained matrix $G \equiv\left[Z, Z^{\dagger}\right]+\Psi \Psi^{\dagger}$ is the generator of the unitary transformations for both $Z$ and $\Psi$, it must obey the commutation relations of the $U(N)$ algebra. In terms of the basis $\left\{I, T^{a}\right\}$ of $U(N)$, the matrix fields $Z$ and $Z^{\dagger}$ can be expanded as

$$
Z=z_{0}+\sum_{a=1}^{N^{2}-1} z_{a} T^{a}, \quad Z^{\dagger}=z_{0}^{\dagger}+\sum_{a=1}^{N^{2}-1} z_{a}^{\dagger} T^{a}
$$


Using these expansions, we can express the constrained matrix as

$$
G^{a}=\operatorname{Tr}\left(G T^{a}\right)=-i f^{a b c} z_{b}^{\dagger} z_{c}+\Psi^{\dagger} T^{a} \Psi,
$$

where $f^{a b c}$ are the structure constants of $S U(N)$, that is, $\left[T^{a}, T^{b}\right]=i f^{a b c} T^{c}$.

After quantisation, the elements of the matrix fields $Z, Z^{\dagger}$ and the vector fields $\Psi$, $\Psi^{\dagger}$ become operators, and satisfy the fundamental commutation relations

$$
\left[\left(Z_{\alpha}\right)_{m n},\left(Z_{\beta}^{\dagger}\right)_{k l}\right]=\delta_{m k} \delta_{n l} \delta_{\alpha \beta}, \quad\left[\left(\Psi_{\alpha}\right)_{m},\left(\Psi_{\beta}^{\dagger}\right)_{n}\right]=\delta_{m n} \delta_{\alpha \beta} .
$$

Furthermore, the expansion modes in the constrained matrix $G$ also become operators. The constrained operators $\widehat{G}^{a}$ satisfy the $S U(N)$ algebra, and can be regarded as the generators of $S U(N)$. Because the generators $T^{a}$ of $S U(N)$ are traceless, the constrained matrix $G$ gives the traceless part of the constraint equation (31). After quantisation, the operators $\hat{G}^{a}$ become the projected operators of the quantum physical states in the matrix model

$$
\widehat{G}^{a} \mid \text { Phys }>=\left(\widehat{G}_{Z}^{a}+\widehat{G}_{\Psi}^{a}\right) \mid \text { Phys }>=0 .
$$

On the other hand, the trace part of (31) produces the following constraint condition of the quantum physical states

$$
\left(\Psi_{n}^{\dagger} \Psi_{n}-2 N \lambda \theta\right) \mid \text { Phys }>=0 .
$$

Since we are considering a matrix model of finite number of particles moving on the two-dimensional sphere, we must also impose the geometrical constraint on the quantum physical states, which maps the manifold parameterised by the coordinates $Z$ to the twosphere $S^{2}$. As mentioned above, $z^{\dagger} z=1$ together with the $U(1)$ gauge transformations of $z$, that is, $z \rightarrow e^{i \alpha} z$, implies that the geometrical condition $n^{a} n^{a}=1$ of $S^{2}$ is satisfied. In our matrix model, this condition becomes

$$
\left[\operatorname{Tr}\left(Z^{\dagger} Z\right)-g\right] \mid \text { Phys }>_{s}=0,
$$

where $g$ is a parameter dependent on the model, and $\mid$ Phys $>_{s}$ stands for the geometrically stable configuration among the quantum physical states.

From the constraint condition (36), we know that the physical states must be the singlet representation of $S U(N)$, of which $\hat{G}^{a}$ are the generators. However, $\hat{G}_{Z}^{a}$ are only realised by the representations arising from products of the adjoint representations of $S U(N)$. Furthermore, $Z_{1}$ and $Z_{2}$ form a spinor, and describe the spin degree of freedom of the particles. So they should appear in pairs in the singlet representation. Therefore, the representations of $\hat{G}_{Z}^{a}$ contain only irreducible representations for which the total number of boxes in the Young diagram is an integer multiple of $2 N$. Since the physical states are invariant under the action of $G_{Z}^{a}+G_{\Psi}^{a}$, the representations of $G_{Z}$ and $G_{\Psi}$ 
must be conjugate to each other so that their product contains the singlet of $S U(N)$. Hence, the irreducible representations of $G_{\Psi}$ must be given by the Young diagram with the number of boxes being a multiple of $2 N$. Following the arguments in [17], from the other constraint condition (37), one knows that the number of boxes equals to the total number of spinor oscillators $\Psi^{\dagger} \Psi$. Thus, we conclude

$$
\lambda \theta=k,
$$

where $k$ is an integer. This relation is the same as the one obtained in [17] for the finite matrix model on the plane. Namely, the level $k$ of the $U(1)$ noncommutative ChernSimons action does not change with the geometry on which the particles move.

In Haldane's description of the quantum Hall effect, spinors are dynamical degrees of freedom of the electrons. On the other hand, it can be easily seen from (36) and (37) that the constraint of the $S U(N)$ invariance (36) is consistent with the vanishing condition of the total $U(1)$ charge (37) only if the quantum physical states are spin singlets.

To summarise, the quantum physical states of our matrix model must possess the following properties. (a) They are the singlet representations of the $S U(N)$ group. (b) They must be the spin singlets, which implies that the same number of spin-up and spin-down components will be present in the quantum physical states, that is, they are the $S U(2)$ invariant states associated with the spin. (c) There are $k N$ number of $\Psi_{1}^{\dagger}$ and $k N$ number of $\Psi_{2}^{\dagger}$ in the quantum physical states, where $\Psi_{1}^{\dagger}$ and $\Psi_{2}^{\dagger}$ form the spinor $\Psi^{\dagger}$. (d) The geometrically stable states among the quantum physical states should satisfy the geometrical constraint condition (38).

Subsequently, we shall determine the quantum physical states of the matrix model, which build up the physical Fock space of the model. Recall that Hamiltonian (32) of the system can be expressed as $\omega \hat{N}_{Z}$ in terms of the number operator $\hat{N}_{Z} \equiv \sum_{m, n} Z_{m n}^{\dagger} Z_{\mathrm{nm}}$ of the spinor oscillators $Z$. From this expression, we know that energy eigenstates will be linear combinations of terms with a fixed number of $Z^{\dagger}$ creation operators acting on the Fock space vacuum $\mid 0>$ defined by

$$
Z_{m n}|0\rangle=\Psi_{n}|0\rangle=0 .
$$

The constraint conditions of quantum physical states require that all physical states must have a fixed number $N k$ of $\Psi_{1}^{\dagger}$ creation operators and the same number of $\Psi_{2}^{\dagger}$ creation operators acting on the Fock vacuum. Furthermore, the number of $Z_{1}^{\dagger}$ creation operators appearing in the physical state should be the same as that of $Z_{2}^{\dagger}$ creation operators. Thus, any physical state which is an energy eigenstate is a sum of terms of the form

$$
\prod_{m=1}^{M}\left(Z_{1}^{\dagger}\right)_{j_{m}}^{i_{m}}\left(Z_{2}^{\dagger}\right)_{j_{m}^{\prime}}^{i^{\prime}} \prod_{n=1}^{N k}\left(\Psi_{1}^{\dagger}\right)_{\iota_{n}}\left(\Psi_{2}^{\dagger}\right)_{l_{n}^{\prime}}|0\rangle
$$

where the upper and lower represent fundamental and anti-fundamental indices of $S U(N)$, respectively. 
Now, the problem is how to construct a singlet of both $S U(N)$ and spin from the equation (41) by contracting all indices with the covariant tensors of $S U(N)$. Since the product of an epsilon tensor with upper indices and one with lower indices may be rewritten as a sum of products of delta functions, we may only need to use one type of epsilon tensors to do the contraction. Let us first of all establish a few lemmas, which are generalisations of the facts given by Hellerman and Raamsolonk in the appendix of their paper [10].

LEMMA 1. Setting $\chi(u, v) \equiv \prod_{i<j}\left(u_{i} v_{j}-u_{j} v_{i}\right)$ where $u$ and $v$ are two components of spinor $z$, that is, $z=\left(\begin{array}{l}u \\ v\end{array}\right)$, we have

$$
\chi(u, v)=\varepsilon^{i_{1} \cdots i_{N}} \prod_{n=1}^{N}\left(u^{N-n} v^{n-1}\right)_{i_{n}},
$$

where we have abbreviated $\varepsilon^{(\tilde{i})_{1} \cdots \bar{i}_{N}} \prod_{n=1}^{N}\left(u^{N-n}\right)_{i_{n}}\left(v^{n-1}\right)_{\bar{i}_{n}}$ as $\varepsilon^{i_{1} \cdots i_{N}} \prod_{n=1}^{N}\left(u^{N-n} v^{n-1}\right)_{i_{n}}$.

Proof: From the definition of $\chi(u, v)$ and its expression (42), one can see that they all are completely antisymmetric, and have the same order in $u$ and $v$ powers. Hence, the definition of $\chi(u, v)$ must equal to its expression up to a numerical factor. Taking a fixed $N$, for example, $N=3$, we can check that the numerical factor is equal to 1 .

LEMMA 2. Any polynomial $D(u, v)=\varepsilon^{i_{1} \cdots i_{N}} \prod_{m=1}^{N}\left(u^{n_{m}} v^{\tilde{n}_{m}}\right)_{i_{m}}$, where $\sum_{i=1}^{N} n_{i}=\sum_{i=1}^{N} \tilde{n}_{i}$, may be written as a sum of terms of the form

$$
F(u, v)=\prod_{n=1}^{N} S_{n}^{c_{n}} \widetilde{S}_{n}^{\tilde{c}_{n}} \chi(u, v)
$$

here $S_{l}=\sum_{i=1}^{N} u_{i}^{l}$ and $\widetilde{S}_{l}=\sum_{i=1}^{N} v_{i}^{l}$. The equality $\sum_{i=1}^{N} n_{i}=\sum_{i=1}^{N} \widetilde{n}_{i}$ is the conclusion of spin singlet, which implies that $\sum_{i=1}^{N} i c_{i}=\sum_{n=1}^{N} i \widetilde{c}_{i}$.

LEMma 3. Suppose that $\Psi_{1}^{\dagger}, \Psi_{2}^{\dagger}$ and $Z_{1}^{\dagger}, Z_{2}^{\dagger}$ be the $N$-dimensional vectors and the $N \times N$ matrices of commuting variables, respectively. Thus, any expression of the form

$$
\begin{aligned}
\mathcal{D}\left(\Psi_{1}^{\dagger}, \Psi_{2}^{\dagger} ; Z_{1}^{\dagger}, Z_{2}^{\dagger}\right) & =\varepsilon^{(\tilde{i})_{1} \cdots(\tilde{i})_{N}} \prod_{l=1}^{N}\left(\Psi_{1}^{\dagger} Z_{1}^{\dagger n_{l}} Z_{2}^{\dagger \tilde{n}_{l}} \Psi_{2}^{\dagger}\right)_{(\tilde{i})_{l}} \\
& \equiv \varepsilon^{i_{1} \cdots i_{N}} \prod_{l=1}^{N}\left(\Psi_{1}^{\dagger} Z_{1}^{\dagger n_{l}} Z_{2}^{\dagger \tilde{n}_{l}} \Psi_{2}^{\dagger}\right)_{i_{l}}
\end{aligned}
$$

may be uniquely expressed as a sum of terms of form

$$
\mathcal{F}\left(\Psi_{1}^{\dagger}, \Psi_{2}^{\dagger} ; Z_{1}^{\dagger}, Z_{2}^{\dagger}\right)=\prod_{i=1}^{N}\left(\operatorname{Tr} Z_{1}^{\dagger i}\right)^{c_{i}}\left(\operatorname{Tr} Z_{2}^{\dagger i}\right)^{\tilde{c}_{i}} \varepsilon^{i_{1} \cdots i_{N}} \prod_{n=1}^{N}\left(\Psi_{1}^{\dagger} Z_{1}^{\dagger N-n} Z_{2}^{\dagger n-1} \Psi_{2}^{\dagger}\right)_{i_{n}} .
$$


The conclusion of this lemma is also true conversely.

The proofs of the lemma 2 and the lemma 3 are completely parallel to those of the fact 2 and the fact 3 provided by Hellerman and Raamsolonk. Here we shall omit them. The above lemmas provide the basis for constructing the quantum physical states of our matrix model.

Now, we return to the contraction of the Fock states (41). The problem is to determine all possible ways of combining the symmetrised anti-fundamentals of $\Psi_{1}^{\dagger}, \Psi_{2}^{\dagger}$ to form a singlet of $S U(N)$. Unlike the finite matrix model of Polychronakos, this singlet of $S U(N)$ is also a spin singlet. Notice that $Z_{1}^{\dagger}, \Psi_{1}^{\dagger}$ should be regarded as the spin-up components and $Z_{2}^{\dagger}, \Psi_{2}^{\dagger}$ as spin-down components. Let us first of all contract $N k$ creation operators $\Psi_{1}^{\dagger}$ with a fixed number of $Z_{1}^{\dagger}$ and $N k$ operators $\Psi_{2}^{\dagger}$ with certain number of $Z_{2}^{\dagger}$ to form a singlet of $S U(N)$. Precisely, we first consider the indices of the $N k$ number of $\Psi_{1}^{\dagger}$. The lower index of $\Psi_{1}^{\dagger}$ must be contracted with either the upper index of $Z_{1}^{\dagger}$ or an epsilon tensor. After the contraction of $\Psi_{1}^{\dagger}$ with one $Z_{1}^{\dagger}$, the resulting object will still have a single lower index, that is, $\left(\Psi_{1}^{\dagger}\right)_{i_{1}}\left(Z_{1}^{\dagger}\right)_{j_{1}}^{i_{1}} \rightarrow\left(\Psi_{1}^{\dagger} Z_{1}^{\dagger}\right)_{j_{1}}$. This lower index may again be contracted with either the upper index of another $Z_{1}^{\dagger}$ or an epsilon tensor. Repeating this process, we conclude that after contracting each $\Psi_{1}^{\dagger}$ with certain number of $Z_{1}^{\dagger}$ the resulting object will only have one free lower index, that is, $\left(\Psi_{1}^{\dagger} Z_{1}^{\dagger n_{1}}\right)_{i_{1}}$. Similarly, we have $\left(\Psi_{2}^{\dagger} Z_{2}^{\dagger \bar{n}_{1}}\right)_{i_{1}}$. However, the indices of $\left(\Psi_{1}^{\dagger} Z_{1}^{\dagger n_{1}}\right)_{i_{1}}$ and $\left(\Psi_{2}^{\dagger} Z_{2}^{\dagger \tilde{n}_{1}}\right)_{\bar{i}_{1}}$ belong to the same particle label since $Z_{1}^{\dagger}$ and $Z_{2}^{\dagger}$ are respectively associated with the spin-up and spin-down components of the particle. This implies that $\Psi_{1}^{\dagger} Z_{1}^{\dagger n_{1}}$ and $\Psi_{2}^{\dagger} Z_{2}^{\dagger \tilde{n}_{1}}$ should appear in one contracted element, that is, $\left(\Psi_{1}^{\dagger} Z_{1}^{\dagger n_{1}} Z_{2}^{\dagger \tilde{n}_{1}} \Psi_{2}^{\dagger}\right)_{(\tilde{i})_{1}}$. The lower indices of $N$ such elements are then contracted with the upper indices of an epsilon tensor to produce the fundamental contraction block

$$
\varepsilon^{i_{1} \cdots i_{N}} \prod_{l=1}^{N}\left(\Psi_{1}^{\dagger} Z_{1}^{\dagger n_{l}} Z_{2}^{\dagger \tilde{n}_{l}} \Psi_{2}^{\dagger}\right)_{i_{l}}
$$

where we have used the abbreviation symbol defined in the lemmas.

Because there are $N k$ number of $\Psi_{1}^{\dagger}$ and $N k$ number of $\Psi_{2}^{\dagger}$ in the quantum physical states, the physical states are composed of $k$ fundamental contraction blocks. So, using the above lemmas, we can write down the minimal basis for the physical energy eigenstates, which is a singlet for both $S U(N)$ and the spin,

$$
\left|\left\{n_{i}\right\},\left\{\tilde{n}_{i}\right\}, k\right\rangle=\varepsilon^{i_{1} \cdots i_{N}} \prod_{l=1}^{N}\left(\Psi_{1}^{\dagger} Z_{1}^{\dagger n_{l}} Z_{2}^{\dagger \tilde{n}_{l}} \Psi_{2}^{\dagger}\right)_{i_{1}}\left(\varepsilon^{i_{1} \cdots i_{N}} \prod_{n=1}^{N}\left(\Psi_{1}^{\dagger} Z_{1}^{\dagger N-n} Z_{2}^{\dagger n-l} \Psi_{2}^{\dagger}\right)_{i_{n}}\right)^{k-1}|0\rangle,
$$

where $\left\{n_{i}\right\}$ and $\left\{\widetilde{n}_{i}\right\}$ satisfy the relation $\sum_{i} n_{i}=\sum_{i} \tilde{n}_{i}$ derived from the spin singlet requirement. On the other hand, $Z_{i}^{\dagger}, i=1,2$, may also be contracted with themselves, leading to terms which are products of $\operatorname{Tr}\left(Z_{1}^{\dagger}\right), \ldots, \operatorname{Tr}\left(Z_{1}^{\dagger N}\right)$ and $\operatorname{Tr}\left(Z_{2}^{\dagger}\right), \ldots, \operatorname{Tr}\left(Z_{2}^{\dagger N}\right)$. By 
means of the Lemma 3, they can be used to build up another set of minimal basis for the physical energy eigenstates

$$
\left|\left\{c_{i}\right\},\left\{\widetilde{c}_{i}\right\}, k\right\rangle=\prod_{i=1}^{N}\left(\operatorname{Tr} Z_{1}^{\dagger i}\right)^{\mathcal{c}_{i}}\left(\operatorname{Tr} Z_{2}^{\dagger i}\right)^{\tilde{c}_{i}}\left(\varepsilon^{i_{1} \cdots i_{N}} \prod_{n=1}^{N}\left(\Psi_{1}^{\dagger} Z_{1}^{\dagger N-n} Z_{2}^{\dagger n-1} \Psi_{2}^{\dagger}\right)_{i_{n}}\right)^{k}|0\rangle
$$

The spin singlet condition of the physical states leads to $\sum_{i=1}^{N} i c_{i}=\sum_{i=1}^{N} i \widetilde{c}_{i}$. Using the Hamiltonian (32), we can easily obtain the energy eigenvalues corresponding to the above bases. The former basis give the energy eigenvalues,

$$
\begin{aligned}
E\left(\left\{n_{i}\right\},\left\{\tilde{n}_{i}\right\}, k\right) & =\omega\left[(k-1) N(N-1)+\sum_{i} n_{i}+\sum_{i} \tilde{n}_{i}\right] \\
& =\omega\left[(k-1) N(N-1)+2 \sum_{i} n_{i}\right],
\end{aligned}
$$

and the latter basis gives rise to

(50) $E\left(\left\{c_{i}\right\},\left\{\tilde{c}_{i}\right\}, k\right)=\omega\left[k N(N-1)+\sum_{i=1}^{N} i c_{i}+\sum_{i=1}^{N} i \tilde{c}_{i}\right]=\omega\left[k N(N-1)+2 \sum_{i=1}^{N} i c_{i}\right]$.

From the expressions (47) and (48) of the minimal bases of the physical energy eigenstates, we find that the physical ground state of the present finite matrix model is given by

$$
|\{0\},\{0\}, k\rangle=\left(\varepsilon^{i_{1} \cdots i_{N}} \prod_{n=1}^{N}\left(\Psi_{1}^{\dagger} Z_{1}^{\dagger N-n} Z_{2}^{\dagger n-1} \Psi_{2}^{\dagger}\right)_{i_{n}}\right)^{k}|0\rangle \equiv L^{\dagger k}|0\rangle
$$

Roughly speaking, after the formal substitutions $\Psi_{1}^{\dagger} \rightarrow 1, \Psi_{2}^{\dagger} \rightarrow 1$ and $Z_{1 j}^{\dagger} \rightarrow A_{1 j}$, $Z_{2 j}^{\dagger} \rightarrow A_{2 j}$ in (51), we get

$$
|0, k\rangle=\left(\varepsilon^{i_{1} \cdots i_{N}} \prod_{n=1}^{N}\left(A_{1}^{\dagger N-n} A_{2}^{\dagger n-1}\right)_{i_{n}}\right)^{k}|0\rangle .
$$

Furthermore, if $u$ and $v$ are regarded as the eigenvalue parameters of $A_{1}$ and $A_{2}$ respectively in the coherent state picture, we find that

$$
\langle u, v \mid 0, k\rangle=\left(\varepsilon^{i_{1} \cdots i_{N}} \prod_{n=1}^{N}\left(u^{N-n} v^{n-1}\right)_{i_{n}}\right)^{k}=\prod_{i<j}\left(u_{i} v_{j}-u_{j} v_{i}\right)^{k},
$$

which is the same as the ground state wave function of the two-dimensional quantum Hall fluid on the spherical geometry [9]. However, as pointed out by Polychronakos [17], the classical value of the inverse filling fraction is shifted quantum mechanically if one uses the finite matrix Chern-Simons theory to describe the fractional quantum Hall states. 
This can be equivalently viewed as renormalising the Chern-Simons coefficient. In fact, this level shift in the matrix Chern-Simons model is the same as the quantum mechanical level shift in the corresponding Chern-Simon theory. The renormalisation of the level for the latter theory has been done in [8] using the BRS invariant regularisation method. This renormalisation leads to the following level shift: $k \rightarrow k+\operatorname{sign}(k) c_{V}$, where $k$ is the bare level parameter and $c_{V}$ the quadratic Casimir in the adjoint representation of the gauge group. As mentioned previously, our finite matrix Chern-Simons model on $S^{2}$ corresponds to the $U(1)$ Chern-Simons theory. Hence, physical states in the matrix model at level $k$ should be identified with the quantum Hall states at the filling fraction $1 /(k+1)$ rather than $1 / k$. That is, for filling fraction $1 /(k+1)$ the Laughlin type wave function in the two-dimensional spherical geometry can be equivalently described by the physical ground state of the finite matrix Chern-Simons model on $S^{2}$.

The reason that the state (51) is regarded as the physical ground state becomes clear from the following discussions. The system considered by us is a priori $2 N(N+1)$ uncoupled oscillators, which consist of $2 N^{2}$ harmonic oscillators from $Z_{1}, Z_{2}$ and $2 N$ harmonic oscillators from $\Psi_{1}, \Psi_{2}$. However, they should be regarded as $N(N+1)$ uncoupled spinor oscillators since $Z=\left(\begin{array}{l}Z_{1} \\ Z_{2}\end{array}\right)$ and $\Psi=\left(\begin{array}{l}\Psi_{1} \\ \Psi_{2}\end{array}\right)$ must be viewed as the spinors describing the particles on $S^{2}$. Furthermore, what couples the spinor oscillators is $N^{2}-1$ constraint equations in the traceless part of the Gauss constraint (31). Effectively, we can describe the system by means of the $N+1$ independent oscillators. All $S U(N)$ invariant states can be spanned by the operators, $Q_{1 n}^{\dagger}=\operatorname{Tr}\left(Z_{1}^{\dagger n}\right), Q_{2 n}^{\dagger}=\operatorname{Tr}\left(Z_{2}^{\dagger n}\right)$ with $n=1,2, \ldots, N$, and $L^{\dagger k}$ acting on the Fock vacuum. However, the spin singlet condition of physical states results in the balancing of the numbers of $\left\{Q_{1 n}^{\dagger}\right\}$ and $\left\{Q_{2 n}^{\dagger}\right\}$ appearing in the physical states. So there are $N$ independent spinor oscillators, which together with the operator $L^{\dagger k}$ provide the $N+1$ independent oscillators. A useful conclusion in mathematics is that the operators $Q_{1 l}^{\dagger}$ and $Q_{2 l}^{\dagger}$ for $l>N$ can be expressed as homogeneous polynomials of total order $l$ in $\left\{Q_{11}^{\dagger}, Q_{12}^{\dagger}, \ldots, Q_{1 N}^{\dagger}\right\}$ and $\left\{Q_{21}^{\dagger}, Q_{22}^{\dagger}, \ldots, Q_{2 N}^{\dagger}\right\}$, respectively, with constant coefficients. Based on this conclusion and the commutation relations between $Z$ and $Z^{\dagger}$, we have $Q_{11} Q_{2 l} L^{\dagger k}|0\rangle=0$, for all $l$. This means that the state $L^{\dagger k}|0\rangle \equiv|0, k\rangle$ is the physical vacuum with respect to all operators $Q_{1 l}$ and $Q_{2 l}$. Equivalently, the Laughlin-type state $|0, k\rangle$ is the physical ground state of our finite matrix model. In the next section, we shall discuss the excitation states produced by acting the creation operators $Q_{1_{1}}^{\dagger}$ and $Q_{2 l_{2}}^{\dagger}$ on the ground state.

\section{QUASI-PARTICLE EXCITATIONS AND HIERARCHY OF QUANTUM HALL FLUIDS}

Following $[17,11]$, the low-lying excitations in our matrix model can be described in terms of quasi-particles and quasi-holes. A quasi-particle state is obtained by peeling a 'particle' from the surface of the Fermi sea. That is, one quasi-particle obtained by 
exciting a 'particle' above the Fermi level by energy amount $n w$ is described by

$$
\begin{aligned}
p_{n}^{1 \dagger}|0, k\rangle= & \left(\varepsilon^{i_{1} \cdots i_{N}} \prod_{m=1}^{N}\left(\Psi_{1}^{\dagger} Z_{1}^{\dagger N-m} Z_{2}^{\dagger m-1} \Psi_{2}^{\dagger}\right)_{i_{m}}\right)^{k-1} \\
& \varepsilon^{i_{1} \cdots i_{N}} \quad\left(\Psi_{1}^{\dagger} Z_{1}^{\dagger N-1+n} Z_{2}^{\dagger 0} \Psi_{2}^{\dagger}\right)_{i_{1}} \prod_{m=2}^{N}\left(\Psi_{1}^{\dagger} Z_{1}^{\dagger N-m} Z_{2}^{\dagger m-1} \Psi_{2}^{\dagger}\right)_{i_{m}}|0\rangle .
\end{aligned}
$$

The quasi-holes correspond to the minimal excitations of the ground state inside the quantum Hall fluid. One quasi-hole excitation is obtained by creating a gap inside the quantum Hall fluid with the energy increase $m \omega$, namely,

$$
\begin{aligned}
h_{m}^{1 \dagger}|0, k\rangle= & \left(\varepsilon^{i_{1} \cdots i_{N}} \prod_{n=1}^{N}\left(\Psi_{1}^{\dagger} Z_{1}^{\dagger N-n} Z_{2}^{\dagger n-1} \Psi_{2}^{\dagger}\right)_{i_{n}}\right)^{k-1} \\
& \varepsilon^{i_{1} \cdots i_{N}} \prod_{n=1}^{m}\left(\Psi_{1}^{\dagger} Z_{1}^{\dagger N-n+1} Z_{2}^{\dagger n-1} \Psi_{2}^{\dagger}\right)_{i_{n}} \prod_{n=m+1}^{N}\left(\Psi_{1}^{\dagger} Z_{1}^{\dagger N-n} Z_{2}^{\dagger n-1} \Psi_{2}^{\dagger}\right)_{i_{n}}|0\rangle .
\end{aligned}
$$

Obviously, $p_{1}^{1 \dagger}=h_{1}^{1 \dagger}$. So there is no fundamental distinction between 'particles' and 'holes' in the matrix model. Similarly, one can describe the quasi-particle excitation $p_{n}^{2 \dagger}$ and quasi-hole excitation $h_{m}^{2 \dagger}$ corresponding to the oscillator field $Z_{2}$. We have

$$
\begin{aligned}
& p_{n}^{2 \dagger}|0, k\rangle=\left(\varepsilon^{i_{1} \cdots i_{N}} \prod_{m=1}^{N}\left(\Psi_{1}^{\dagger} Z_{1}^{\dagger N-m} Z_{2}^{\dagger m-1} \Psi_{2}^{\dagger}\right)_{i_{m}}\right)^{k-1} \\
& \varepsilon^{i_{1} \cdots i_{N}} \prod_{m=1}^{N-1}\left(\Psi_{1}^{\dagger} Z_{1}^{\dagger N-m} Z_{2}^{\dagger m-1} \Psi_{2}^{\dagger}\right)_{i_{m}}\left(\Psi_{1}^{\dagger} Z_{1}^{\dagger 0} Z_{2}^{\dagger N-1+n} \Psi_{2}^{\dagger}\right)_{i_{1}}|0\rangle,
\end{aligned}
$$

and

$$
\begin{aligned}
h_{m}^{2 \dagger}|0, k\rangle= & \left(\varepsilon^{i_{1} \cdots i_{N}} \prod_{n=1}^{N}\left(\Psi_{1}^{\dagger} Z_{1}^{\dagger N-n} Z_{2}^{\dagger n-1} \Psi_{2}^{\dagger}\right)_{i_{n}}\right)^{k-1} \\
& \varepsilon^{i_{1} \cdots i_{N}} \prod_{n=1}^{N-m}\left(\Psi_{1}^{\dagger} Z_{1}^{\dagger N-n} Z_{2}^{\dagger n-1} \Psi_{2}^{\dagger}\right)_{i_{n}} \prod_{n=N-m+1}^{N}\left(\Psi_{1}^{\dagger} Z_{1}^{\dagger N-n} Z_{2}^{\dagger n} \Psi_{2}^{\dagger}\right)_{i_{n}}|0\rangle .
\end{aligned}
$$

Although all these excitations are fundamental ones in the finite matrix model, they can not be regarded directly as the physical low-lying excitations. The physical exciting states must obey the constraint condition, which is nothing but the spin singlet condition of the physical states. Using the lemmas shown by us in the previous section, one finds that all fundamental excitations can be equivalently expressed in the following form

$$
P_{n_{1}, n_{2}}^{\dagger}|0, k\rangle=\prod_{j=1}^{N}\left(\operatorname{Tr} Z_{1}^{\dagger j}\right)^{c_{j}}\left(\operatorname{Tr} Z_{2}^{\dagger j}\right)^{\tilde{c}_{j}}|0, k\rangle=\prod_{j=1}^{N}\left(Q_{1 j}^{\dagger}\right)^{c_{j}}\left(Q_{2 j}^{\dagger}\right)^{\tilde{c}_{j}}|0, k\rangle,
$$


where, $n_{1}=\sum_{i=1}^{N} i c_{i}$ and $n_{2}=\sum_{i=1}^{N} i \widetilde{c}_{i}$. Indeed, they are exciting states with respect to the Laughlin-type ground state $|0, k\rangle$. The physical excitation states can be constructed by using the expression (56) of the fundamental excitations, together with the spin singlet condition of the physical states.

Following Haldane [9], we can construct the collective ground state of the twodimensional quantum Hall fluid from our matrix model by condensing the quasi-particle and quasi-hole excitations. The condensation here means that the physical state including the excitations becomes the Laughlin-type fluid state, like the physical ground state $|0, k\rangle$. This Laughlin-type fluid state is given by

$$
\left|0, p_{1}, k\right\rangle=\left(\varepsilon^{i_{1}, \ldots, i_{N_{1}}} \prod_{n=1}^{N_{1}}\left(P^{1+N_{1}-n} P^{2 \nmid n-1}\right)_{i_{n}}\right)^{p_{1}}|0, k\rangle,
$$

where $N_{1}=N / p_{1}+1, N$ are divisible by $p_{1}$, and $P^{1+i}, P^{2+i}$ stand for $P_{i, 0}^{\dagger}, P_{0, i}^{\dagger}$, which are defined by (56). In fact, the condition $N_{1}=N / p_{1}+1$ is required by the fact that if one uses the operators $\left\{Q_{1 n}^{\dagger}\right\}$ and $\left\{Q_{2 n}^{\dagger}\right\}$ as a set of independent creation operators, the condition of operator powers $n \leqslant N$ must be satisfied, otherwise, the constructed creation operators will not be independent. Using the lemmas in the previous section, one can easily check that the excitation fluid state indeed satisfies the spin singlet condition of the physical states in the matrix model.

We can also construct the excitation states of the two-dimensional excitation fluid by the procedure similar that used in the construction of the excitation states of $|0, k\rangle$. To do this it is more convenient to use the latter basis for the Fock space of the matrix model. Introducing operators $P_{s}^{1 \dagger j}=\sum_{n=1}^{N_{1}} P_{n}^{1 \dagger j}$ and $P_{s}^{2 \nmid j}=\sum_{n=1}^{N_{1}} P_{n}^{2 \dagger j}$, we can write the excitation states of the excitation fluid as

$$
\left|\left\{c_{1 i}\right\},\left\{\tilde{c}_{1 i}\right\}, p_{1}, k\right\rangle=\prod_{j=1}^{N_{1}}\left(P_{s}^{1 \dagger j}\right)^{c_{1 j}}\left(P_{s}^{2 \nmid j}\right)^{\tilde{c}_{1 j}}\left|0, p_{1}, k\right\rangle .
$$

Although these states do not generally satisfy all the constraint conditions of the physical states in the matrix model, they are useful intermediate states for constructing the physical ground state of the excitation fluid in the next level. Here, the further constraint condition of the physical excitation states is the spin singlet condition. One can get the next level of the Laughlin-type fluid state by further condensing the 'quasi-particle' and 'quasi-hole' excitations of the excitation fluid. Furthermore, the process of constructing the two-dimensional excitation fluids in our matrix model can be iterated, which leads to a hierarchy of two-dimensional quantum Hall fluid states. We here give the result of the iterated quantum Hall states

$$
\left|0, p_{m}, \ldots, p_{1}, k\right\rangle=\prod_{q=1}^{m}\left(\varepsilon^{i_{1}, \ldots, i_{N_{q}}} \prod_{n=1}^{N_{q}}\left(P_{q-1}^{1 \dagger N_{q}-n} P_{q-1}^{2 \nmid n-1}\right)_{i_{n}}\right)^{p_{q}}|0, k\rangle
$$


where the iterated relation is given by $p_{q}\left(N_{q}-1\right)+N_{q+1}=N_{q-1}$ with $N_{q}=0$ for $q>m$ and $N_{0}=N$. The fundamental excitation operators of the excitation fluids, $P_{m}^{1+j} \equiv P_{j, 0}^{(m)}$ and $P_{m}^{2 \dagger j} \equiv P_{0, j}^{(m)}$, are determined by the equations

$$
P_{n_{1}, n_{2}}^{(m)}\left|0, p_{m}, \ldots, p_{1}, k\right\rangle=\prod_{j=1}^{N_{m}}\left(P_{s, m-1}^{1 \dagger j}\right)^{c_{j}}\left(P_{s, m-1}^{2 \dagger j}\right)^{\tilde{c}_{j}}\left|0, p_{m}, \ldots, p_{1}, k\right\rangle
$$

where $n_{1}=\sum_{j=1}^{N_{m}} j c_{j}$ and $n_{2}=\sum_{j=1}^{N_{m}} j \widetilde{c}_{j}$. The symmetric operators in (60) are defined as $P_{s, m-1}^{1 \dagger j}=\sum_{i=1}^{N_{m}}\left(P_{m-1}^{1 \dagger j}\right)_{i}$ and $P_{s, m-1}^{1 \dagger j}=\sum_{i=1}^{N_{m}}\left(P_{m-1}^{1 \dagger j}\right)_{i}$. In fact, in the process of constructing the hierarchy of the two-dimensional quantum Hall fluids, we have also constructed all quasi-particle and quasi-hole excitations of the quantum Hall excitation fluids.

Haldane's idea on constructing the hierarchy of the fluid states is to consider a field strength slightly different from its parent one. The hierarchical fluid states are the low-energy states corresponding to this field strength, which can be considered as being derived from the Laughlin-type fluid state associated with the parent field strength through imbalancing quasi-particle and quasi-hole excitations. In our matrix model, the physical states $\left|0, p_{m}, \ldots, p_{1}, k\right\rangle$ correspond to Haldane's low-energy states. Physically, the imbalance of quasi-particle and quasi-hole excitations leads to the degeneracy of the lowest Landau level state in the hierarchical fluids so that

$$
2 S+1=k(N-1)+N_{1}+1,
$$

where $N_{1}$ is the contribution from the imbalance of the quasi-particle and quasi-hole excitations in the background of the quantum Hall fluid of electrons. Such $N_{1}$ can be obtained by solving the iterated equations $p_{q}\left(N_{q}-1\right)+N_{q+1}=N_{q-1}$. The result is

$$
\begin{aligned}
N_{1}=\frac{N}{p_{1}+\left(1 / p_{2}+\cdots+\left(1 / p_{m}\right)\right)}+\frac{1}{\left(p_{1}+\left(1 / p_{2}+\cdots+\left(1 / p_{m}\right)\right)\right)} \\
{\left[p_{1}-\frac{1}{\left(p_{2}+\left(1 / p_{3}+\cdots+\left(1 / p_{m}\right)\right)\right)}\left[\cdots\left[p_{m-2}-\frac{p_{m-1}-1}{p_{m-1}+\left(1 / p_{m}\right)}\right]\right]\right] . }
\end{aligned}
$$

The filling factor means the occupation factor of the lowest Landau level. For general hierarchical fluid states, the imbalancing of excitations in the background of the quantum Hall fluid of electrons can be affected by the imbalance of excitations of the excitation fluids. This results in a different $N_{1}$. The spins of electrons in the hierarchical fluid state are given by $(1 / 2) \widetilde{k}(N-1)+N_{1} / 2$, where we have applied the level shifting $k \rightarrow \widetilde{k}=k+1$ while keeping the number of electrons $N$ intact. So we obtain the filling factor of the $m$-th hierarchical fluid state in the thermodynamic limit

$$
\nu=\lim _{N \rightarrow \infty} \frac{N}{\widetilde{k}(N-1)+N_{1}+1}=\frac{1}{\widetilde{k}+\left(1 / p_{1}+\left(1 / p_{2}+\cdots+\left(1 / p_{m}\right)\right)\right)} .
$$


Since the hierarchical quantum Hall fluid states are derived directly from the condensation of the excitations in the quantum Hall fluid of electrons, we find the energies of these hierarchical fluid states by using the Hamiltonian expression of the matrix model. Explicitly,

$$
H\left|0, p_{m}, \ldots, p_{1}, k\right\rangle=\omega\left[k N(N-1)+\sum_{q=1}^{m} p_{q} N_{q}\left(N_{q}-1\right)\right]\left|0, p_{m}, \ldots, p_{1}, k\right\rangle .
$$

Substituting the iterated equations $p_{q}\left(N_{q}-1\right)+N_{q+1}=N_{q-1}$ into the above equation, we obtain the energy of the $m$-th hierarchical fluid state

$$
E\left(p_{m}, \ldots, p_{1}, k\right)=\omega\left[k N(N-1)+N N_{1}\right]
$$

The term involving $N_{1}$ in the energy comes from the contribution of condensing the excitations. This implies that these hierarchical fluid states are the sub-stable states of the matrix model since their energies are higher than the energy of the parent fluid state, that is, the physical ground state of the matrix model. It should be emphasised that the hierarchical quantum Hall fluids are dynamically formed by condensing the 'quasiparticle' and 'quasi-hole' excitations level by level. In other words, such hierarchical fluid states consistently exist in our matrix model without the need of any modifications of the model.

\section{SUMmarY AND OUTLOOK}

If one considers particle motion on the two-sphere in a radial monopole magnetic field, the configurations of the particle coordinates can not be smoothly defined globally over the entire $S^{2}$ due to the singularity resulting from the Dirac monopole. The effective action of the system can be described in a singularity-free way by using a nontrivial bundle over $S^{2}$, which can be obtained by the Hopf fibration with base $S^{2}$. The existence of the Dirac monopole in the 2-dimensional quantum Hall system makes the particle coordinates on the two-sphere noncommutative. The appearance of such monopole also results in the irreducible representations of the $S U(2)$ algebra satisfied by the particle coordinates on $S^{2}$ to be truncated, leading to the fuzzy $S^{2}$ geometry. We have explicitly shown that the noncommutative structure of the fuzzy $S^{2}$ appears indeed in the Haldane model of the quantum Hall effect by restricting to the lowest Landau level state. In order to establish the noncommutative field theory for the quantum Hall system, we have provided the Hopf mapping for the fuzzy $S^{2}$, that is, (14) and (15). This mapping plays an essential role in our model. The Hopf fibration of $S^{2}$ can be regarded as a principal fibre bundle with the base space $S^{2}$ and the $U(1)$ structure group. This $U(1)$ gauge group is iterated in the formulation of the noncommutative field theory for the quantum Hall fluids on $S^{2}$. This implies that our model is a finite matrix version of the $U(1)$ noncommutative 
Chern-Simons theory on $S^{2}$. It involves matrix vector fields of spinors, different from the finite matrix model proposed by Polychronakos. In fact, the Hopf mapping of the fuzzy manifolds related to the second Hopf map is very important for the description of the quantum Hall effect on $S^{4}$ and the open two-brane in M theory $[21,6]$. It should be interesting to further recognise the mathematical implication of Hopf mappings between fuzzy manifolds and their applications in physics.

Hellerman and Raamsdonk [10] had speculated that the secondly-quantised field theoretical description of the quantum Hall fluids for various filling fractions is given by a regularised matrix version of the noncommutative $U(1)$ Chern-Simons theory on the plane. We have attempted to establish the secondly-quantised field theoretical description of the quantum Hall fluids on the two-sphere for various filling fractions, and have determined some essential physical properties of the quantum Hall fluids. Moreover, we have determined the complete set of the physical quantum states for the finite matrix model on $S^{2}$, and shown the correspondence between the physical ground state of our model and the Laughlin-type wave function. Although such a determination of physical states is a generalisation of the work of Hellerman and Raamsdonk for the finite matrix model on the plane, it is nontrivial because the spinor matrix vector fields are included in our finite matrix model.

It is an interesting conclusion of our work that the hierarchical Hall fluid states can be dynamically generated in the finite matrix model on $S^{2}$. The formation of these hierarchical Hall fluid states originates from the condensing of excitations of the quantum Hall fluids level by level. This dynamical mechanism is consistent with the original idea of Haldane. In the process of constructing the hierarchical Hall fluid states, we have found the explicit forms of 'quasi-particle' and 'quasi-hole' excitations in each level of the hierarchy. We believe that these results are helpful for studying the correlation and interaction behaviour of the excitations in the quantum Hall effect.

\section{REFERENCES}

[1] I.J.R. Aitchison, 'Berry phases, magnetic monopoles, and Wess-Zumino terms or how the skyrmion got its spin', Acta Phys. Polon. B 18 (1987), 207-235.

[2] A.P. Balachandran, K.S. Gupta and S. Kurkcuoglu, 'Edge currents in non-commutative Chern-Simons theory from a new matrix model', (e-print hep-th/0306255).

[3] A.P. Balachandran, 'TASI lectures at Yale unversity', (1985).

[4] T. Banks, W. Fischler, S.S. Shenker and L. Susskind, ' $M$ theory as a matrix model: A conjecture.', Phys. Rev. D 55 (1997), 5112-5128.

[5] B.A. Bernevig, J.H. Brodie, L. Susskind and N. Toumbas, 'How Bob Laughlin tamed the giant graviton from Taub-NUT space', J. High Energy Phys. (2001), 26.

[6] Y.X. Chen, 'Rigid open membrane and non-abelian non-commutative Chern-Simons theory', (e-print hep-th/0211156).

[7] G.V. Dunne, R. Jackiw and C.A. Trugenberger, " "Topological" (Chern-Simons) quantum mechanics', Phys. Rev. D 41 (1990), 661-666. 
[8] G. Giavarini, C.P. Martin and F.Ruiz Ruiz, 'Chern-Simons theory as the large-mass limit of topologically massive Yang-Mills theory', Nuclear Phys. B 381 (1992), 222-280.

[9] F.D.M. Haldane, 'Fractional quantization of the Hall effect: A hierarchy of incompressible quantum fluid states', Phys. Rev. Lett. 51 (1983), 605-608.

[10] S. Hellerman and M.V. Raamsdonk, 'Quantum Hall physics equals noncommutative field theory', J. High Energy Phys. 10 (2001), 18.

[11] L. Jonke and S. Meljanac, 'Finite Chern-Simons matrix model - algebraic approach', $J$. High Energy Phys. 8 (2002), 13.

[12] D. Karabali and B. Sakita, 'Chern-Simons matrix model: coherent states and relation to Laughlin wave functions', Phys. Rev. B 64 (2001), 8.

[13] R.B. Laughlin, 'Anomalous quantum Hall effect: an incompressible quantum fluid with fractionally charged excitations', Phys. Rev. Lett. 50 (1983), 1395-1398.

[14] F. Lizzi, P. Vitale and A. Zampini, 'The fuzzy disc', (e-print hep-th/0306247).

[15] J. Madore, 'The fuzzy sphere', Classical Quantum Gravity 9 (1992), 69-87.

[16] B. Morariu and A.P. Polychronakos, 'Finite noncommutative Chern-Simons with a Wilson line and the quantum Hall effect', J. High Energy Phys. 7 (2001), 15.

[17] A.P. Polychronakos, 'Quantum Hall states as matrix Chern-Simons theory', J. High Energy Phys. 4 (2001), 20.

[18] M. Stone, 'Born-Oppenheimer approximation and the origin of Wess-Zumino terms: Some quantum-mechanical examples', Phys. Rev. D 33 (1986), 1191-1194.

[19] L. Susskind, 'The quantum Hall fluid and non-commutative Chern-Simons theory', (e-print hep-th/0101029).

[20] T.T. Wu and C.N. Yang, 'Concept of nonintegrable phase factors and global formulation of gauge fields', Phys. Rev. D12 (1975), 3845-3857.

[21] S.C. Zhang and J.P. Hu, 'A four-dimensional generalization of the quantum Hall effect', Science 294 (2001), 823-828.

Zhejiang Institute of Modern Physics

Zhejiang University

Hangzhou 310027

Peoples Republic of China

and

Department of Mathematics

The University of Queensland

Queensland 4072

Australia

e-mail: yxchen@zimp.zju.edu.cn

\author{
Department of Mathematics \\ The University of Queensland \\ Queensland 4072 \\ Australia \\ Department of Mathematics \\ The University of Queensland \\ Queensland 4072 \\ Australia \\ e-mail: yzz@maths.uq.edu.au
}

